\section{Geschätztes Standardwerk in neuer Auflage}

S echs Jahre nach Erscheinen der Vorauflage liegt nun das „Gutachten des Hals-Nasen-Ohren-Arztes " in einer vollständig überarbeiteten Neufassung vor. Dieses weit über den HNO-Bereich hinaus geschätzte Standardwerk wird von Ärzten, BGs und Sozialgerichten gleichermaßen seit nunmehr fast 40 Jahren als Begleiter bei der Gutachtenerstellung und -bewertung genutzt.

In die Neuauflage wurden verschiedene, in den vergangenen Jahren aktualisierte Verordnungen und Leitlinien wie z.B. das Königsteiner Merkblatt -, aber auch die Liste der Berufskrankhei- ten und diverse Formulare aufgenommen. Veränderungen im Stellenwert zu begutachtender Krankheitsbilder, wie z.B. der Lärmschwerhörigkeit, die in den letzten Jahren auf Grund vorbeugender Maßnahmen an Bedeutung verloren hat, wird ebenso Rechnung getragen wie neuen Erkenntnissen zum HWS-Schleudertrauma. Mehr als 100 Fallbeispiele aus Gutachtertätigkeit und Rechtsprechung tragen wesentlich zum praktischen Nutzen bei. Den Abschluss bilden ein umfangreiches Literaturverzeichnis sowie Hinweise auf weiterführende Begutachtungsliteratur. Müßig zu erwäh-

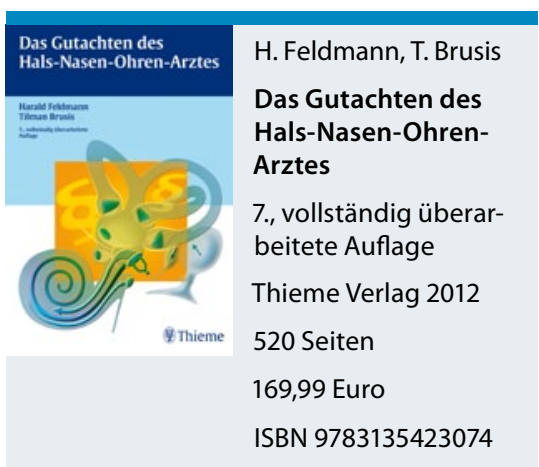

nen, dass das Buch in der aktualisierten Form in den Bücherschrank eines jeden gehört, der sich mit gutachterlicher Tätigkeit befasst.

Prof.Dr.G. Grevers

\title{
Was Stimmtherapeuten wissen sollten
}

D as hier vorgestellte Lehrbuch ist ein Wegweiser im weiten Feld der Stimmtherapie und liefert kompakt aufbereitete Fachkenntnisse: Anatomie, Physiologie und Pathophysiologie, Anamnese und Diagnostik, gängige Therapiekonzepte im Überblick und Vergleich und - in fünf Therapiebausteinen strukturiert - einen methodenübergreifenden und zeitgemäßen Therapieansatz. $\mathrm{Zu}$ al- len Themen gibt es weiterführende Literaturempfehlungen.

Ergänzt in der 5. Auflage sind drei neu entwickelte Behandlungsmethoden, die inklusive Vergleich und Bewertung im Buch vorgestellt werden: Das Funktionale Stimmtraining (Erlanger Modell), das Lee Silverman Voice Treatment und die Resonanzmethode nach Gall.

\section{Aktualisiertes Wissen über Schwindel- und Gleichgewichtsstörungen}

\footnotetext{
$\int$ chwindel ist keine Diagnose, sondern $S$ ein unspezifisches fachübergreifendes Symptom für verschiedene Erkrankungen unterschiedlicher Ätiologien. Dieses klinisch orientierte Buch unterstützt Fachärzte, die Patienten mit Schwindel versorgen.

Die wichtigsten Schwindelsyndrome sind übersichtlich dargestellt, die Untersuchungsgänge ausführlich geschildert und mit Video- und Fotomaterial illustriert. Die einheitliche Kapitelstruktur lehnt sich konsequent an die Praxis an: Anamnese, Klinik und Verlauf, Pathophysiologie und therapeutische Prinzipien, pragmatische Therapie, Wirksam-
}

keit sowie Differenzialdiagnose und klinische Probleme.

Die 2. Auflage bildet die Fortschritte der letzten Jahre auf dem Gebiet der Schwindel- und Gleichgewichtsstörungen und deren Therapien komprimiert und verständlich ab: neue Untersuchungsmethoden und Bildgebungsverfahren, aktuelle Therapiemöglichkeiten zu allen Krankheitsbildern, ein eigenes Kapitel zur medikamentösen Therapie, plus DVD mit zahlreichen neuen Filmsequenzen mit Untersuchungsgängen und typischen Befunden.

Langjährige Behandlungs- und Forschungserfahrungen fließen in dieses

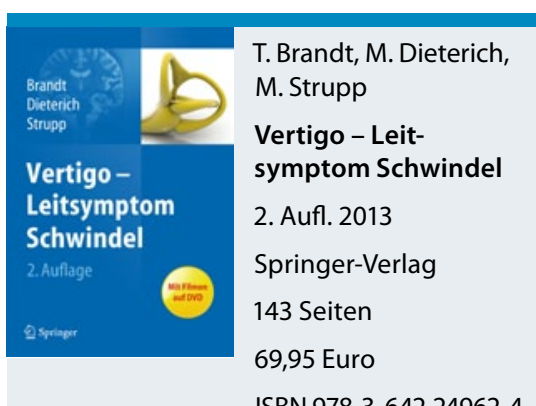

ISBN 978-3-642-24962-4

Buch ein, insbesondere die Arbeit des Autorenteams an der interdisziplinären Schwindelambulanz des Integrierten Forschungs- und Behandlungszentrums (IFB) für Schwindel, Gleichgewichtsund Augenbewegungsstörungen in München. 